\title{
Downlink Steered Space-Time Spreading For Multi-Carrier Transmission Over Frequency Selective Channels
}

\author{
Nazar Sahal, Mohammed El-Hajjar and Lajos Hanzo \\ School of Electronics and Computer Science, University of Southampton, SO17 1BJ, UK. \\ Email: $\{$ meh05r,1h\}@ecs.soton.ac.uk, \\ http: //www-mobile.ecs. soton.ac.uk
}

\begin{abstract}
This paper presents a novel amalgam of Steered Space-Time Spreading (SSTS) and Orthogonal Frequency Division Multiple Access (OFDMA) designed for attaining both spatial diversity gain and beamforming gain for transmission over OFDM-symbol-invariant frequency selective channels. We propose a flexible technique for increasing the number of users beyond the number of chips in the spreading sequence employed by the SSTS scheme with the aid of the multiple carriers of OFDMA, which requires an extended bandwidth. However, employing a separate low-complexity SSTS detector combined with another separate OFDMA detector is potentially less complex than a single detector designed for detecting all the users supported in a single domain, regardless whether the singledomain Multi-User Detector (MUD) is an SSTS or OFDMA MUD. This is because the MUD's complexity tends to increase exponentially with the number of users detected. The SSTSOFDMA system is benchmarked against its counterpart using S-depth Frequency Domain (FD) repetition, which increases the FD diversity order at the cost of reducing the overall throughput by a factor of S. Fortunately, a similar FD diversity gain may be achieved without a factor-S reduction in the throughput, when using FD spreading and assigning all the superimposed FD spreading codes to the same user.
\end{abstract}

\section{INTRODUCTION}

Multiple-Input Multiple-Output (MIMO) systems attain a higher capacity than their single-input single-output counterparts [1,2]. A substantial diversity gain has been attained by Alamouti's witty design [3], which was later generalised in [4] as Space-Time Block Coding (STBC). Inspired by the philosophy of STBCs, Hochwald et al. [5] proposed the transmit diversity concept known as Space-Time Spreading (STS) for the DownLink (DL) of Wideband Code Division Multiple Access (WCDMA) [6].

Furthermore, in recent years numerous research contributions have appeared on the topic of Multi-Carrier Direct Sequence Code Division Multiple Access (MC DS-CDMA), which constitutes an attractive scheme [6-9] based on a combination of DS-CDMA and Orthogonal Frequency Division Multiplexing (OFDM) [10]. In [11], a generalised MC DSCDMA scheme was proposed that includes the subclasses of both multitone and orthogonal MC DS-CDMA as special cases. In [12] MC DS-CDMA using STS was investigated in the context of broadband communications over frequency selective fading channels.

The financial support of Vodafone under the auspices of the Dorothy Hodgkin Postgraduate Award as well as that of the European Union and the EPSRC, UK is gratefully acknowledged.
On the other hand, beamforming [13] constitutes an effective technique of reducing the Multiple-Access Interference (MAI), where the antenna gain is increased in the direction of the desired user, whilst reducing the gain towards the interfering users. Furthermore, in order to achieve additional performance gains, beamforming [13] has been combined with STBC to attain a higher Signal-to-Noise Ratio (SNR) gain [14]. A further generalised multi-functional MIMO scheme was proposed in [15] in order to design a system having a high multiplexing gain, a high diversity gain as well as a beamformer gain.

In $[16,17]$ a Steered STS (SSTS) scheme was proposed for the sake of attaining both diversity gain as well as beamforming gain. The SSTS scheme of [17] employs generalised MC DS-CDMA combined with spreading in both the TimeDomain (TD) and Frequency-Domain (FD) for the sake of increasing the attainable system load either by increasing the number of users supported or their individual bitrates. Moreover, in [18] the authors proposed a MIMO-OFDM system for transmission over frequency selective channels.

In this contribution we amalgamate the merits of SSTS and Orthogonal Frequency Division Multiple Access (OFDMA) for the sake of achieving spatial diversity gain as well as beamforming gain, when communicating over OFDM-symbolinvariant frequency selective channels. We demonstrate that the number of users supported and/or their bitrates can be increased with the aid of combining both STS TD spreading and OFDMA. The resultant SSTS-OFDMA system is benchmarked against its counterpart using S-depth FD repetition, which increases the FD diversity order. In all cases, an OFDM-symbol-invariant frequency selective channel is assumed. Therefore, the rationale and novelty of the paper can be summarised as follows

1) We amalgamate the benefits of STS and beamforming for the sake of achieving both DL transmit-diversity gain as well as a beamforming gain.

2) SSTS is combined with OFDMA in order to strike a flexible trade-off between the number of users supported and their individual bitrates, while maintaining a good performance over a frequency selective channel.

3) The comparison of the proposed SSTS-OFDMA system and an SSTS assisted S-depth FD-repetition aided OFDMA (SD-FDRA-OFDMA) scheme reveals that the $S D$-FDRA-OFDMA aided system has an S-order FD diversity gain, while commensurately reducing the achiev- 


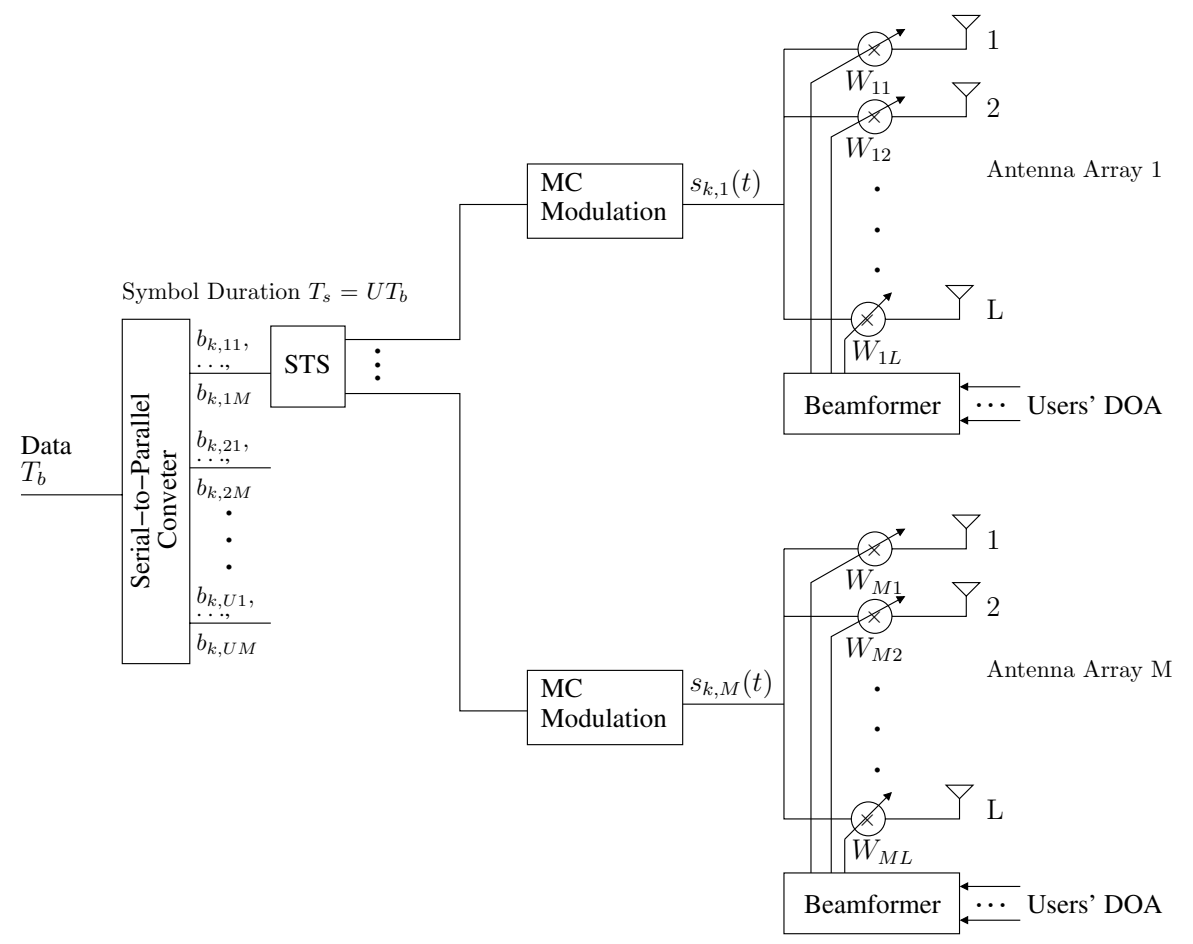

Fig. 1. The proposed system model.

\section{able throughput.}

The rest of the paper is organised as follows. In Section II the transmitter and receiver model of the proposed system communicating over an OFDM-symbol-invariant frequency selective channel is detailed. In Section III the concept of normalised system load is introduced and a technique striking a flexible trade-off between the number of users and their bitrates is described. We characterise the achievable system performance in Section IV and finally we conclude in Section $\mathrm{V}$.

\section{SYSTEM MODEL}

The block diagram of the proposed DL scheme is illustrated in Figure 1. According to Figure 1, a block of $U M$ data bits, each having a bit duration of $T_{b}$, is serial-to-parallel converted to $U$ parallel sub-blocks. The new bit duration of each sub-stream, which is referred to as the symbol duration becomes $T_{s}=U T_{b}$. Following the STS procedure described in [5], where the transmitted signal is spread to $M$ transmit antennas with the aid of the $M$ orthogonal spreading codes of $\left\{c_{k, 1}, c_{k, 2}, \ldots, c_{k, M}\right\}, k=1,2, \ldots, K$ is the number of users, the $U M$ outputs of the $U$ STS blocks is multicarrier modulated employing OFDM and using Binary Phase Shift Keying (BPSK) and then forwarded to the transmitter's DL beamformer. The symbol duration of the STS signal is $M T_{s}$ and the discrete period of the orthogonal STS codes is $M T_{s} / T_{c}=M N_{e}$, where $N_{e}=T_{s} / T_{c}$ and $T_{c}$ represents the chip duration of the orthogonal STS codes. Finally, according to the $k$ th user's channel information, the $U M$ signals of the $k$ th user are weighted by the transmit weight vector $\mathbf{w}_{m}^{(k)}$ determined for the $k$ th user, which is generated for the $m$ th Antenna Array (AA).

The baseband representation of the $k$ th user's SSTS signal on the $n$th subcarrier is given by

$$
s_{k}^{(n)}=\sqrt{\frac{2 E_{b}^{(n)}}{U L} \frac{1}{M M}} \mathbf{W}_{\mathbf{k}}^{(\mathbf{n})} \mathbf{B}_{k} \mathbf{C}_{k},
$$

where $E_{b}^{(n)}$ represents the transmitted power of subcarrier $n$, the factor $L$ in the denominator is due to beamforming distributing the DL transmit power to $L$ elements and the factor $M M$ in the denominator suggests that the STS scheme using $M$ transmit antennas and $M$ orthogonal STS spreading codes distributes its power proportionately in space and time. The spreading sequence matrix $\mathbf{C}_{k}$ is a $(U M \times U)$-element matrix whose entries represent the spreading codes of the different AAs. Additionally, the AA weight matrix $\mathbf{W}_{k}^{(n)}$ can be expressed as

$$
\mathbf{W}_{k}^{(n)}=\left[\begin{array}{cccc}
\mathbf{w}_{0}^{k, n} & \mathbf{0} & \ldots & \mathbf{0} \\
\mathbf{0} & \mathbf{w}_{1}^{k, n} & \ldots & \mathbf{0} \\
\vdots & \vdots & \ddots & \vdots \\
\mathbf{0} & \mathbf{0} & \ldots & \mathbf{w}_{M-1}^{k, n}
\end{array}\right]
$$

where $\mathbf{w}_{m}^{k, n}$ is the $(L \times 1)$-element weight vector of the $k$ th user in the $m$ th AA, $m \in[0, M-1]$, and of the $n$th subcarrier, while the vector $\mathbf{0}$ is an $(L \times 1)$-element zero vector. The vector $\mathbf{B}_{k}$ is given by

$$
\mathbf{B}_{k}=\left[\mathbf{B}_{k 1}^{T}, \mathbf{B}_{k 2}^{T}, \ldots, \mathbf{B}_{k U}^{T}\right]
$$


where $\mathbf{B}_{k u}^{T}$ represents the transmit vector of the $k$ th user generated from the $u$ th STS block, where $u \in[1, U]$.

The $N_{f}$ sub-carriers employed to modulate the OFDM signal are orthogonal, satisfying the relationship of:

$$
\frac{1}{T} \int_{0}^{T}\left(e^{2 j \pi f_{m} t}\right)^{*}\left(e^{2 j \pi f_{n} t}\right) d t=\left\{\begin{array}{ll}
1, & m=n \\
0, & m \neq n
\end{array} .\right.
$$

The SSTS-OFDMA symbol can be expressed as

$$
\mathbf{s}_{k}(t)=\left[\mathbf{s}_{k}^{(1)}(t), \mathbf{s}_{k}^{(2)}(t), \ldots, \mathbf{s}_{k}^{(n)}(t), \ldots, \mathbf{s}_{k}^{\left(N_{f}\right)}(t)\right] .
$$

Assuming that a sufficiently long Cyclic Prefix (CP) was concatenated by the OFDM scheme, so that the CP length is higher than the maximum delay spread, the channel will appear circular to the symbols [19] and hence we can represent the received SSTS OFDM symbol as

$$
\mathbf{r}_{k}=\operatorname{FFT}\left[\operatorname{IFFT}\left(\mathbf{s}_{k}\right) \otimes \mathbf{H}+\hat{\mathbf{N}}\right],
$$

where FFT represents the Fast Fourier Transform, IFFT the inverse FFT based modulation and $\otimes$ denotes the circular convolution operation. Furthermore, $N$ represents the Additive White Gaussian Noise (AWGN) and $\mathbf{H}$ denotes the multipath Channel's Impulse Response (CIR). Thus $\mathbf{r}_{k}$ can be represented by

$$
\mathbf{r}_{k}=\mathbf{s}_{k} \odot \operatorname{FFT}(\mathbf{H})+\mathbf{N}=\mathbf{s}_{k} \odot \mathbf{h}_{k}+\mathbf{N},
$$

where $\odot$ represents a point-wise multiplication operation and $\mathbf{N}=\operatorname{FFT}(\hat{\mathbf{N}})$. Furthermore, we have

$$
\begin{gathered}
\mathbf{h}_{k}=\left[\mathbf{h}_{k}^{(1)}, \mathbf{h}_{k}^{(2)}, \ldots, \mathbf{h}_{k}^{(n)}, \ldots, \mathbf{h}_{k}^{\left(N_{f}\right)}\right] \text {, where } \\
\mathbf{h}_{k}^{(n)}=\left[\begin{array}{ccc}
\mathbf{h}_{k 0,0}^{(n)} & \ldots & \mathbf{h}_{k 0,(M-1)}^{(n)} \\
\vdots & \vdots & \ddots \\
\mathbf{h}_{k(U-1), 1}^{(n)} & \ldots & \mathbf{h}_{(U-1)(M-1)}^{(n)}
\end{array}\right],
\end{gathered}
$$

and $\mathbf{h}_{k u, m}^{(n)}$ represents the $k$ th user's CIR for the $u$ th STS block, the $n$th subcarrier and the $m$ th AA, where $u \in[1, U]$ and $m \in[1, M]$, which can be written as

$$
\mathbf{h}_{k u, m}^{(n)}=\mathbf{a}_{k u, m}^{(n)}=\left[a_{k u, m 0}^{(n)}, \ldots, a_{k u, m(L-1)}^{(n)}\right]^{T},
$$

where $a_{k u, m l}^{(n)}$ is the CIR encountered by the $u$ th subcarrier of the $k$ th user and the $l$ th element of the $m$ th AA. Based on the assumption that the beamforming elements are separated by half a wavelength, we have

$$
\mathbf{a}_{k u, m}^{(n)}=\alpha_{k u, m}^{(n)} \mathbf{d}^{(k)}
$$

where $\alpha_{k u, m}^{(n)}$ is the Rayleigh fading envelope, $\mathbf{d}^{(k)}=$ $\left[1, \exp \left(j\left[\pi \sin \left(\psi^{(k)}\right)\right]\right), \ldots, \exp \left(j\left[(L-1) \pi \sin \left(\psi^{(k)}\right)\right]\right)\right]^{T}$ and $\psi^{(k)}$ is the $k$ th user's Direction of Arrival (DOA).

We assume that the channel is temporally slowly fading and that each subcarrier encounters independent and identical Rayleigh distribution. Additionally, we assume that the $K$ users' signals expressed in the form of (1) are transmitted synchronously over the DL channels. Based on the orthogonality of the STS codes, we multiply both sides of (7) with the spreading code matrix $\mathbf{C}_{1}$ of the desired user, that is of user 1 in this case. In this scenario, the Maximum Ratio Combining (MRC) based transmit beamformer, which constitutes an effective solution to maximising the achievable antenna gain in the direction of the desired user, namely user 1 in this case, is the optimum beamformer. Let $\mathbf{w}_{m}^{k}=\mathbf{d}^{(k)}$ in (10), then the resultant decision variable can be expressed as

$$
\mathbf{Z}_{1}^{(n)}=\sqrt{\frac{2 E_{b}^{(n)}}{U L} \frac{1}{M M}} \tilde{\mathbf{h}}_{1}^{(n)} \mathbf{B}_{1}^{(n)}+\mathbf{N C}_{1}^{\dagger},
$$

where $\dagger$ represents the conjugate transpose operation and $\tilde{\mathbf{h}}_{1}^{(n)}=L \times\left[\alpha_{1 u, 0}^{(n)}, \ldots, \alpha_{1 u,(M-1)}^{(n)}\right]$. Finally, the STS despreading scheme of [5] is employed for the sake of estimating the transmitted source bits.

\section{INCREASING THE NUMBER OF USERS SUPPORTED BY USING OFDMA}

The STS scheme advocated is capable of supporting a maximum number of users, which is equal to the STS TD spreading factor employed. The spreading is carried out by the STS scheme in the TD and the SSTS scheme is combined with OFDMA for the sake of increasing the number of users, while communicating over an OFDM-symbol-invariant frequency selective channel. Additionally, let us define the term "system load" $L_{s}$ as follows:

$$
L_{s}=\frac{K}{S_{f} \times K_{S G}},
$$

where $K$ is the number of users, $S_{f}$ is the SSTS Spreading Factor (SF) and $K_{S G}$ denotes the number of subcarrier groups, which is defined as the ratio of the total number of subcarriers $K_{s}$ and the number of subcarriers per user $K_{u}$. Therefore, the maximum system load of SSTS without an error floor is unity and increasing the number of users beyond that would imply that different users will share the same orthogonal TD spreading code, which would lead to intolerable inter-user interference and a concomitant error floor.

Additionally, the proposed system employs OFDMA for supporting multiple users, where the different users transmit their data on different orthogonal subcarriers. Our aim is to keep the system load defined in Equation (12) less than or equal to unity, so that the system's BER performance does not suffer from any error floor. Employing OFDMA implies that the different users will be distinguishable by their orthogonal subcarriers. To elaborate further on the technique of users' separation employing TD STS spreading and OFDMA, let us consider the following algorithm, where the total OFDMA bandwidth is considered to be fixed:

- When the number of users $K$ is less than the SSTS scheme's SF $S_{f}$, different orthogonal spreading codes are assigned to different SSTS users and OFDMA is employed for mitigating the effects of the frequency selective channels and for increasing the users' bitrate. 
- When the number of users $K$ is increased beyond the SSTS scheme's SF, the users having different STS spreading codes share the same subcarriers in the OFDMA scheme. Users sharing the same SSTS spreading code transmit their data on different OFDMA subcarriers.

- The maximum attainable system load $L_{s}$ is equal to unity according to the definition in Equation (12). For example, if the OFDMA system employs $K_{s}=1024$ subcarriers and the SSTS scheme employs a SF of $S_{f}=8$, then the system load will vary as follows:

1) If the total number of users communicating at the same time is $K=4$, then all the $K_{s}=1024$ subcarriers may be allocated to all the users and the system load becomes $L_{S}=\frac{K}{S_{f} \times K_{S G}}=\frac{4}{8 \times 1}=0.5$. Then all users transmit 1024 bits per OFDM symbol in a BPSK modulated system.

2) If the total number of users communicating at the same time is $K=16$, then $1024 /(16 / 8)=512$ different OFDMA subcarriers will be allocated to each user and the number of subcarrier groups in this case is $K_{S G}=\frac{1024}{512}=2$. Thus, the system load becomes $L_{s}=$ $\frac{16}{8 \times 2}=1$. The number of bits/user/OFDMA symbol then becomes 512 for a BPSK modulated system.

3 ) If the total number of users communicating at the same time is $K=27$, then the number of subcarrier groups in this case is $K_{S G}=4$, where 256 subcarriers will be assigned to each group. The system load becomes $L_{s}=\frac{27}{8 \times 4}=0.84375$. The number of bits/user/OFDMA symbol then becomes 256 for a BPSK modulated system.

\section{Performance Results}

In this section, we consider the DL SSTS-OFDMA system employing an $(M \times L)$-element transmit $\mathrm{AA}$ and a single receive antenna, where $M=2$ transmit AAs are employed. An OFDM-symbol-invariant frequency selective channel is considered and a slow temporal Rayleigh fading channel having a normalised Doppler frequency of $f_{D}=0.001$ is considered. Additionally, perfect channel estimation is assumed at the receiver and BPSK modulation is employed. As mentioned previously, the total OFDMA bandwidth is considered to be fixed.

Figure 2 portrays the Bit Error Ratio (BER) of the proposed SSTS system employing $L=1$ beamforming element per AA, which corresponds to no beamforming and a SF of 8 for supporting a single user. In Figure 2 we also plotted the STS performance in a flat Rayleigh fading channel as a benchmarker. The performance of the SSTS-OFDMA scheme does not depend on the number of subcarriers, since the channel is OFDM-symbol-invariant, when OFDMA is capable of transforming the dispersive channel into a non-dispersive one.

Furthermore, in Figure 3 a comparison between the BER performance of the SSTS-OFDMA scheme supporting a variable number of users is presented. When the FD userseparation technique of Section III is not activated, the users

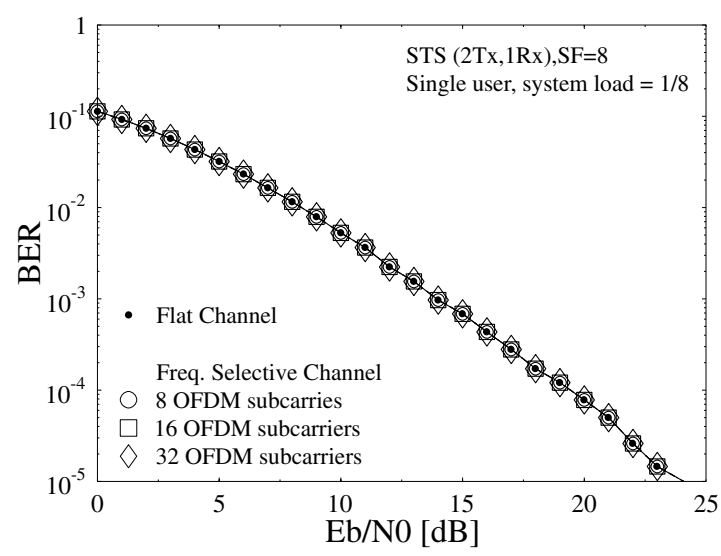

Fig. 2. BER performance of the proposed SSTS-OFDMA system communicating over both a flat Rayleigh fading channel and a frequency selective channel, while having a system load of $1 / 8$ and varying the number of subcarriers per user.

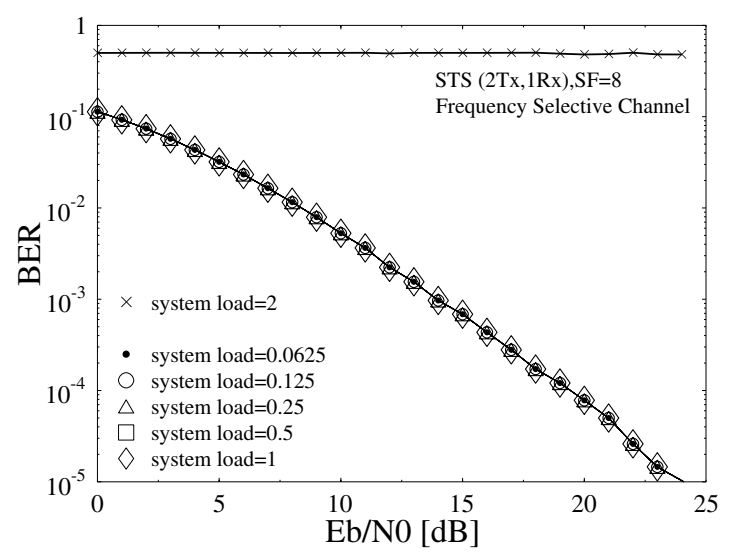

Fig. 3. BER performance of the proposed SSTS-OFDMA system in a frequency selective channel, while varying the system load as well as the number of subcarriers per user.

sharing the same STS codes have a $50 \%$ BER due to the presence of inter-user interference, when the system load $L_{s}=$ 2. By contrast, when employing the SSTS-OFDMA-based technique of Section III, the users' performance is identical to a single user's performance, since no inter-user interference in present, because the STS codes are orthogonal. For the scenarios supporting system loads of $0.0625,0.125,0.25,0.5$ and 1, each user's BER performance is identical, provided that the SSTS-OFDMA-based technique of Section III is activated.

In Figure 4 we plotted the BER performance of the proposed SSTS-OFDMA system employing beamforming having $L=$ 1,2 and 4 elements per AA and a variable system load. As shown in the figure, when the number of beam-steering elements $L$ increases, the achievable BER performance substantially improves. Additionally, the same BER performance is attained for the system loads of 0.5 and 1 , since there is no inter-user interference when the FD user-separation technique of Section III is activated.

A comparison between the BER performance of the SSTS- 


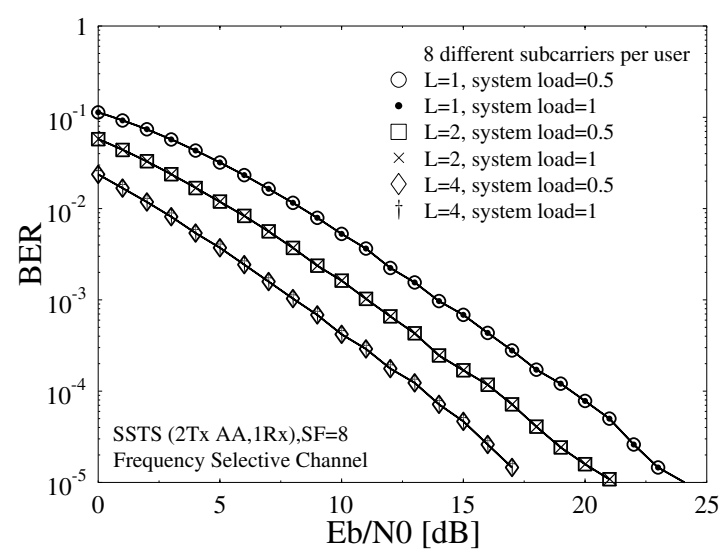

Fig. 4. BER performance of the proposed SSTS-OFDMA system employing a variable number of $L$ beamforming elements per AA in a frequency selective channel, while varying the system load.

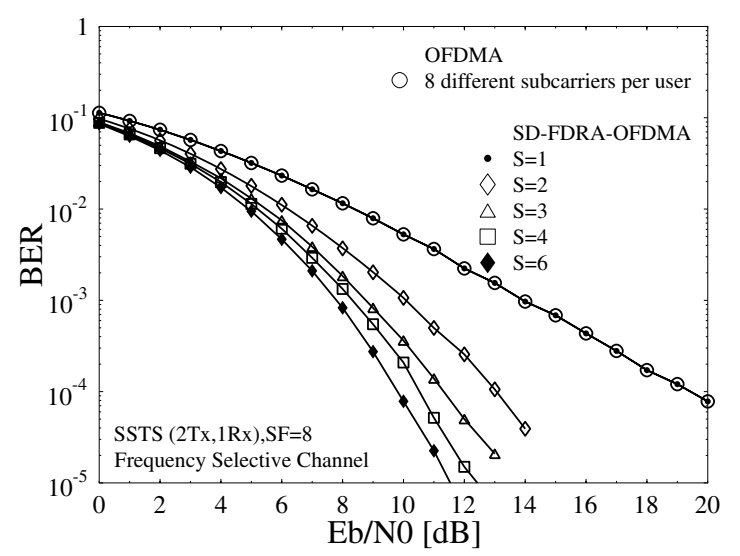

Fig. 5. BER performance comparison of the proposed SSTS-OFDMA aided system and of the SSTS assisted SD-FDRA-OFDMA system employing an S-depth subcarrier repetition.

OFDMA scheme and the SSTS-aided SD-FDRA-OFDMA scheme is shown in Figure 5. An S-depth subcarrier repetition scheme is employed by the OFDMA scheme in a similar manner to that in [12], where in [12] the S-depth FD-repetition was combined with MC CDMA. As shown in Figure 5, the SSTS-aided SD-FDRA-OFDMA system attains an S-order diversity gain. As expected, the SD-FDRA-OFDMA system's BER performance is similar to that of the SSTS-OFDMA scheme when $\mathrm{S}=1$. By contrast, as $\mathrm{S}$ increases, the SD-FDRAOFDMA aided system's BER performance improves owing to its increased diversity order at the cost of a commensurately reduced throughput.

\section{CONCLUSion}

In this paper we proposed a system that amalgamates the advantages of spatial diversity as well as beamforming gain based on the principle of SSTS in order to enhance the attainable performance of OFDMA in OFDM-symbolinvariant frequency selective channels. The proposed system attains a substantial spatial diversity gain as a benefit of using multiple AAs combined with $L$ th-order beamforming for the sake of achieving a high SNR gain, when compared to conventional systems employing a single element per AA, i.e. no beamforming. OFDMA was also invoked for increasing the number of users beyond the SF $S_{f}$ of the STS scheme, while communicating over OFDM-symbol-invariant frequency selective channels. Additionally, the SSTS-OFDMA aided system was compared to its counterpart using S-depth FDrepetition, which attains an S-order diversity gain at the cost of an S-fold throughput reduction.

\section{REFERENCES}

[1] G. Foschini and M. Gans, "On limits of wireless communications in a fading environment when using multiple antennas," Kluwer Academic Publishers, Wireless Personal Communications, pp. 311-335, 1998.

[2] E. Telatar, "Capacity of multi-antenna Gaussian channels," European Transactions on Telecommunications, vol. 10, pp. 585-595, Nov./Dec. 1999.

[3] S. Alamouti, "A simple transmit diversity technique for wireless communications," IEEE Journal on Selected Areas in Communications, vol. 16, no. 8, pp. 1451-1458, 1998.

[4] V. Tarokh, H. Jafarkhani, and A. Calderbank, "Space-time block codes from orthogonal designs," IEEE Transactions on Information Theory, vol. 45, no. 5, pp. 1456-1467, 1999.

[5] B. Hochwald, T. Marzetta, and C. Papadias, "A transmitter diversity scheme for wideband CDMA systems based on space-time spreading," IEEE Journal on Selected Areas in Communications, vol. 19, no. 1, pp. 48-60, 2001.

[6] L. Hanzo, L.-L. Yang, E.-L. Kuan, and K. Yen, Single and Multi-Carrier DS-CDMA: Multi-user Detection, Space-Time Spreading, Synchronisation, Networking and Standards. Chichester, England: John Wiley and Sons Ltd and IEEE Press, NY, USA, 2003.

[7] E. A. Sourour and M. Nakagawa, "Performance of orthogonal multicarrier CDMA in a multipath fading channel," IEEE Transactions on Communications, vol. 44, pp. 356-367, March 1996.

[8] L. Vandendorpe, "Multitone spread spectrum multiple access communications system in a multipath Rician fading channel," IEEE Transactions on Vehicular Technology, vol. 44, pp. 327-337, May 1995.

[9] S. Hara and R. Prasad, "Overview of multicarrier CDMA," IEEE Communications Magazine, vol. 35, pp. 126-133, Dec. 1997.

[10] L. Hanzo, M. Muenster, B. Choi, and T. Keller, OFDM and MC-CDMA for broadband multi-user communications, WLANs and broadcasting. Chichester, UK: Wiley, 2003.

[11] L.-L. Yang and L. Hanzo, "Performance of generalized multicarrier DS-CDMA over Nakagami-m fading channels," IEEE Transactions on Communications, vol. 50, pp. 956-966, June 2002.

[12] L.-L. Yang and L. Hanzo, "Space-time spreading assisted broadband MC DS-CDMA," in IEEE 55th Vehicular Technology Conference, vol. 4, pp. 1881-1885, May 2002.

[13] J. Blogh and L. Hanzo, Third-generation systems and intelligent wireless networking: smart antennas and adaptive modulation. John Wiley \& Sons - IEEE Press, 2002.

[14] G. Jongren, M. Skoglund, and B. Ottersten, "Combining beamforming and orthogonal space-time block coding," IEEE Transactions on Information Theory, vol. 48, pp. 611-627, Mar. 2002.

[15] M. El-Hajjar and L. Hanzo, "Layered steered space-time codes and their capacity," Electronics Letters, vol. 43, pp. 680-682, June 2007.

[16] R. A. Soni, R. M. Buehrer, and R. D. Benning, "Intelligent antenna system for CDMA2000," IEEE Signal Processing Magazine, vol. 19, pp. 54-67, July 2002.

[17] M. El-Hajjar, R. Tee, B. Hu, L.-L. Yang, and L. Hanzo, "Downlink steered space-time spreading assisted generalised multicarrier DSCDMA using sphere-packing-aided multilevel coding," in IEEE Vehicular Technology conference, Sep-Oct 2007.

[18] R. Doostnejad, T. J. Lim, and E. Sousa, "On spreading codes for the down-link in a multiuser MIMO/OFDM system," IEEE 58th Vehicular Technology Conference, vol. 1, pp. 498-502, Oct 2003.

[19] N. Ahmed and R. Baraniuk, "Asymptotic performance of transmit diversity via OFDM for multipath channels," in IEEE Global Telecommunications Conference GLOBECOM, vol. 1, pp. 691-695, Nov 2002. 